\title{
Erratum: Sorkin-Johnston vacuum for a massive scalar field in the 2D causal diamond [Phys. Rev. D 100, 045007 (2019)]
}

\author{
Abhishek Mathur and Sumati Surya \\ (Received 28 July 2021; published 1 October 2021)
}

DOI: $10.1103 /$ PhysRevD.104.089901

We correct a simulation error in our paper which led to the incorrect conclusion in Sec. V that the causal set SorkinJohnston Wightman function $W_{S J}^{c}$ is incompatible with the Rindler Wightman function $W_{m}^{\text {rind }}$ in the corner of a 2D Minkowski diamond for a scalar field with large mass (with respect to the size of the diamond). Instead we find that it is as compatible as the mirror Wightman function $W_{m}^{\text {mirror }}$, which we had shown is compatible with $W_{S J}^{c}$ for all masses. As we discuss now, this seeming compatibility with $W_{m}^{\text {rind }}$ can be traced to the fact that for our simulations, $W_{m}^{\text {rind }} \sim W_{m}^{\text {mirror }}$ for large mass. Note that this does not affect the analytic results of our paper for small mass, nor its broader conclusions which remain unchanged.

The error in our paper was due to using incompatible coordinates to simulate $W_{m}^{\text {rind }}$ which led to the erroneous Fig. 17. This was used to suggest that only $W_{m}^{\text {mirror }}$ is compatible with $W_{S J}^{c}$. We find instead, that there is a correlation between $W_{S J}^{c}$ and $W_{m}^{\text {rind }}$, but further analysis shows that $W_{m}^{\text {rind }}$ and $W_{m}^{\text {mirror }}$ themselves become indistinguishable for larger $m$. To flesh this out we have explored a larger range of masses than discussed in our paper. Figures 1 and 2 show that while the correlation of $W_{S J}^{c}$ and $W_{m}^{\text {mirror }}$ remains largely unchanged with mass, that with $W_{m}^{\text {rind }}$ increases with mass. This can be traced to the increased correlation between $W_{m}^{\text {rind }}$ and $W_{m}^{\text {mirror }}$ with mass as shown in Fig. 3. This in turn is related to the dominance of $W_{m}^{\text {mink }}$ in the expressions for $W_{m}^{\text {rind }}$ and $W_{m}^{\text {mirror }}$ [Eqs. (103) and (104) in our paper] for large mass, as shown in Fig. 4.

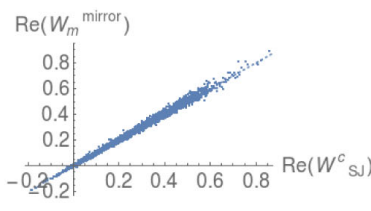

(a) $m=0.1$

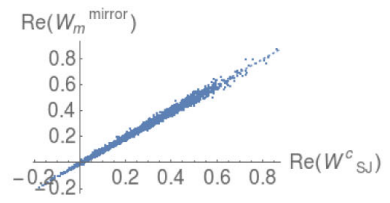

(e) $m=1$

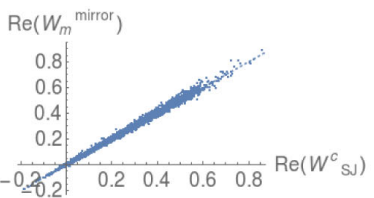

(b) $m=0.2$

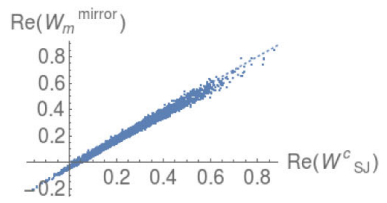

(f) $m=2$

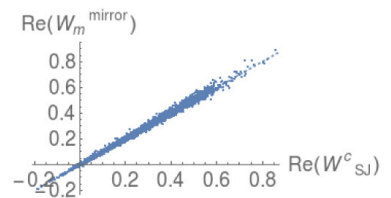

(c) $m=0.3$

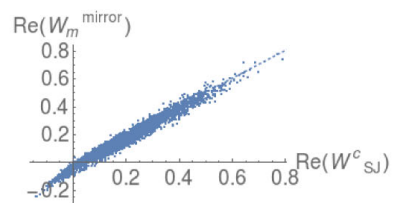

(g) $m=5$

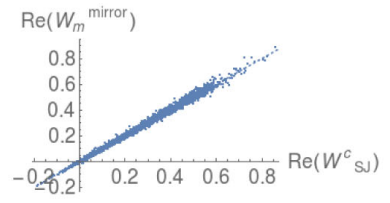

(d) $m=0.4$

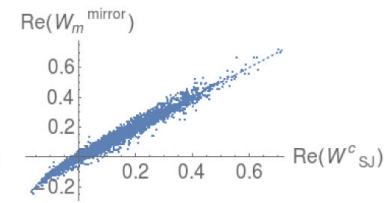

(h) $m=8$

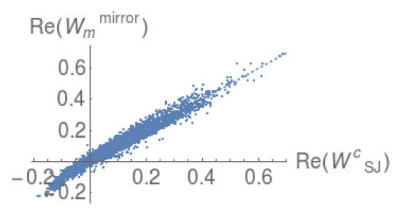

(i) $m=10$

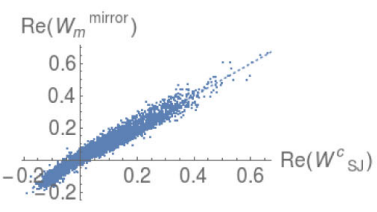

(j) $m=12$

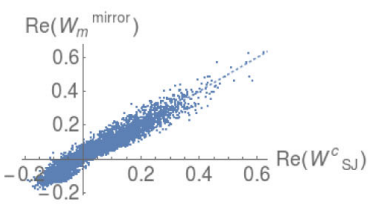

(k) $m=15$

FIG. 1. A correlation plot of the real parts of $W_{S J}^{c}$ vs $W_{m}^{\text {mirror }}$ in the left-hand corner of the 2D causal diamond for a range of masses. The diagonal is denoted by a dotted line. As is evident, the correlation remains largely unchanged with mass. The increase in scatter with mass is related to the fact that the density of sprinkling is left unchanged.

Published by the American Physical Society under the terms of the Creative Commons Attribution 4.0 International license. Further distribution of this work must maintain attribution to the author(s) and the published articles title, journal citation, and DOI. 


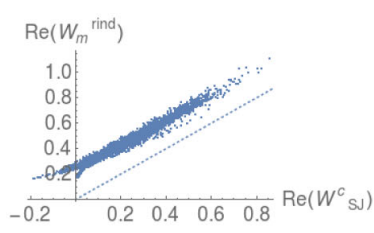

(a) $m=0.1$

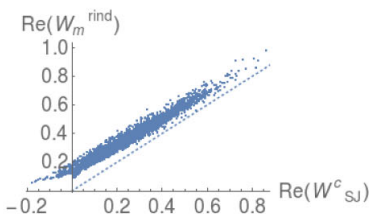

(e) $m=1$

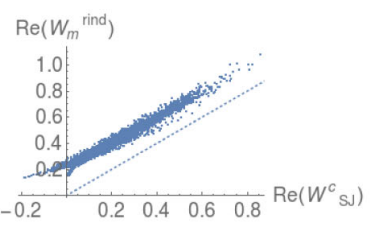

(b) $m=0.2$

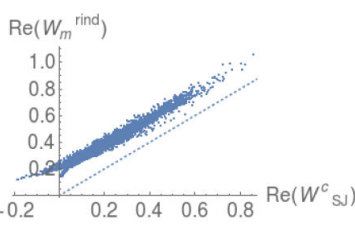

(c) $m=0.3$

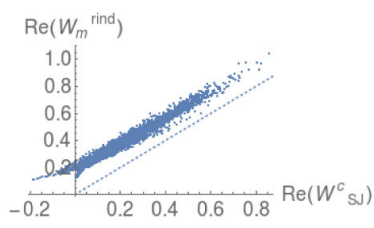

(d) $m=0.4$

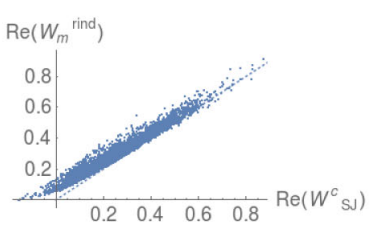

(f) $m=2$

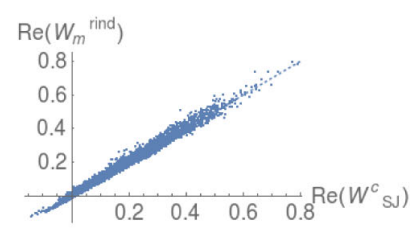

(g) $m=5$

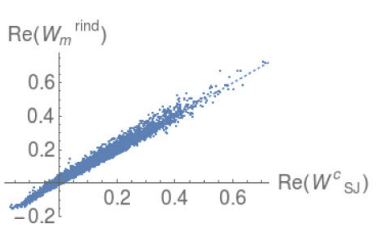

(h) $m=8$

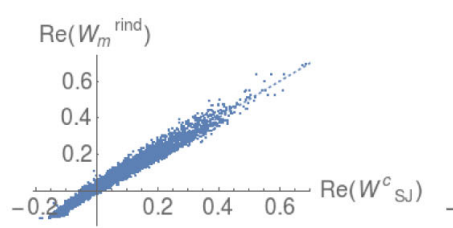

(i) $m=10$

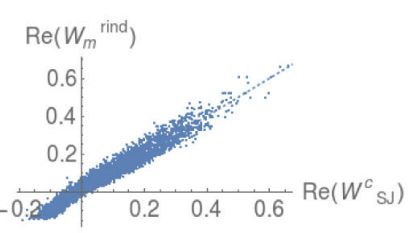

(j) $m=12$

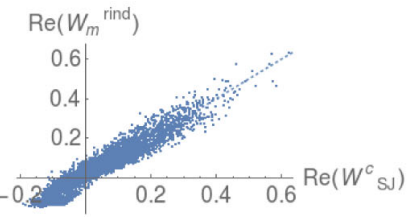

(k) $m=15$

FIG. 2. A correlation plot of the real parts of $W_{S J}^{c}$ vs $W_{m}^{\text {rind }}$ for the same range of masses. For small masses, the correlation is poor but improves with mass.

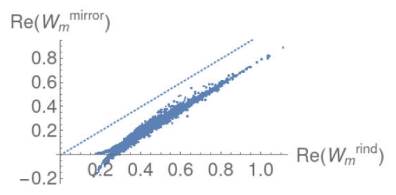

(a) $m=0.1$

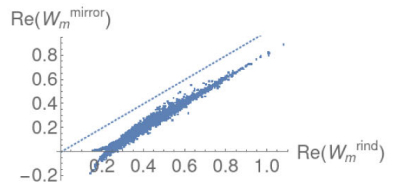

(b) $m=0.2$

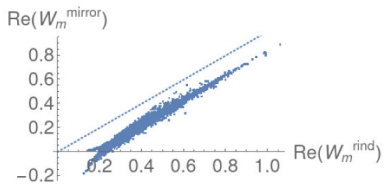

(c) $m=0.3$

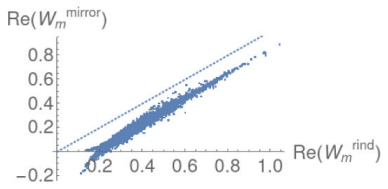

(d) $m=0.4$

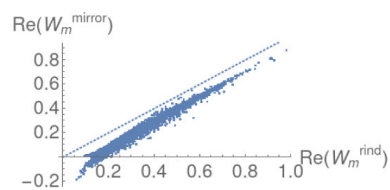

(e) $m=1$

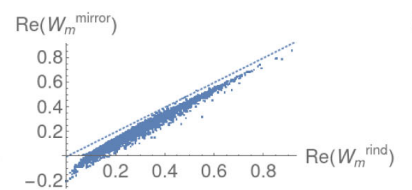

(f) $m=2$

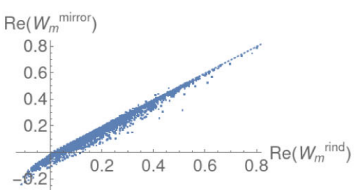

(g) $m=5$

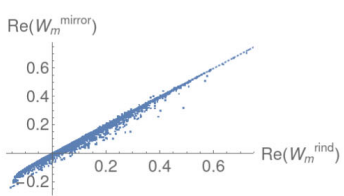

(h) $m=8$

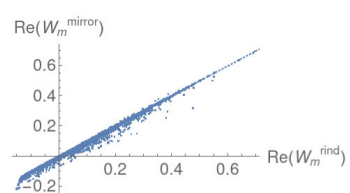

(i) $m=10$

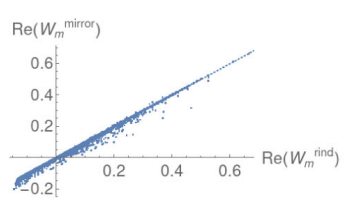

(j) $m=12$

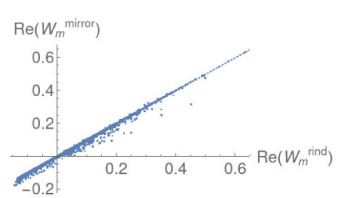

(k) $m=15$

FIG. 3. A correlation plot of the real parts of $W_{m}^{\text {mirror }}$ vs $W_{m}^{\text {rind }}$ for the same range of masses. For small masses, the correlation is poor but improves with mass.

The difference is not captured by our current causal set simulations for which $N \sim 10000$ elements are sprinkled into the larger diamond (of height $2 \sqrt{2}$ ) to give $\sim 118$ elements in the corner diamond (of height $\sim 0.3$ ). Whether this "degeneracy" in choice of vacuum is broken with significantly larger simulations is a question we leave to future investigations.

It was recently brought to our notice that the $m=10$ case was also studied in [1]. 


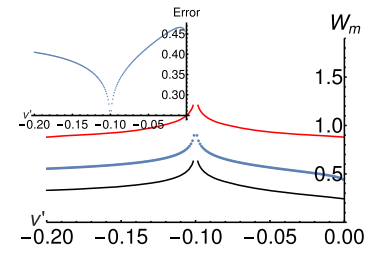

(a) $m=0.1$

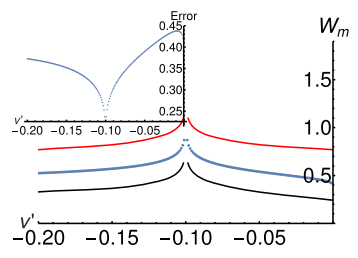

(b) $m=0.2$

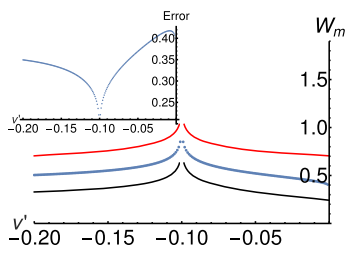

(c) $m=0.3$

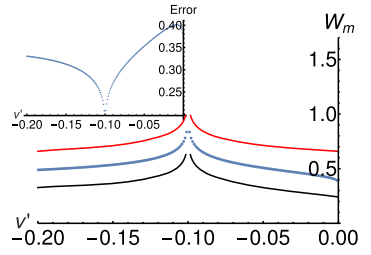

(d) $m=0.4$

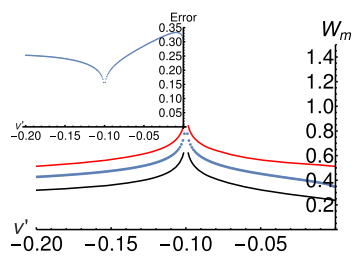

(e) $m=1$

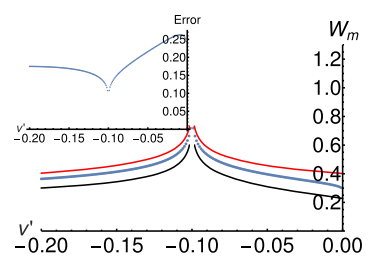

(f) $m=2$

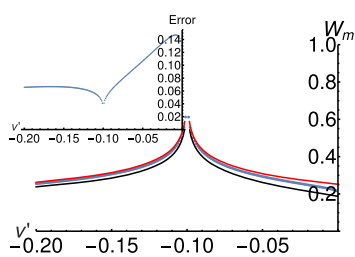

(g) $m=5$

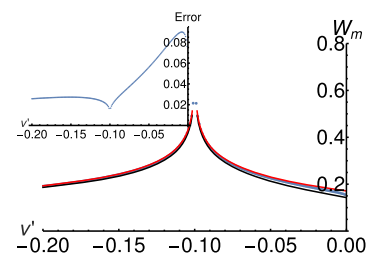

(h) $m=8$

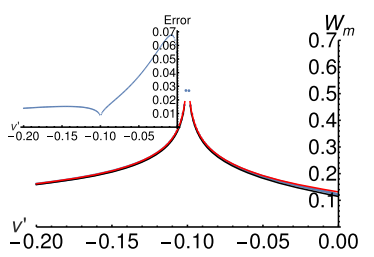

(i) $m=10$

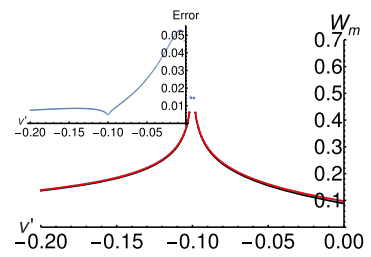

(j) $m=12$

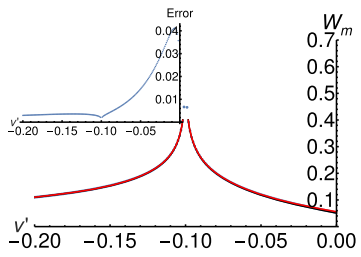

(k) $m=15$

FIG. 4. Real parts of $W_{m}^{\text {mink }}$ (red), $W_{m}^{\text {rind }}$ (blue), and $W_{m}^{\text {mirror }}$ (black) for a pair of points $(u=0.1, v=-0.1)$ and $\left(u^{\prime}=0.11, v^{\prime}\right)$ with varying $v^{\prime}$. As the mass increases all three converge to a common value. To make the comparison explicit, the inset figure shows the relative error between the real parts of $W_{m}^{\text {rind }}$ and $W_{m}^{\text {mirror }}$ as a function of $v^{\prime}$.

\section{ACKNOWLEDGMENTS}

We are grateful to Hans Muneesamy for pointing out the simulation error in our paper. His master's thesis [2] also discusses the $m=1$ and $m=10$ cases. S. S. is supported in part by a Visiting Fellowship at the Perimeter Institute. Research at Perimeter Institute is supported in part by the Government of Canada through the Department of Innovation, Science and Economic Development Canada and by the Province of Ontario through the Ministry of Colleges and Universities.

[1] Y. K. Yazdi, A spacetime approach to defining vacuum states and entropy, MS thesis, University of Waterloo, 2013.

[2] H. Muneesamy, Quantum field theory and entanglement entropy in a 2D scalar causal set, MS thesis, Imperial College, London, 2021. 